\title{
Heart Rate Variability in Young Women with Polycystic Ovary Syndrome
}

\author{
Aylin Yildirir, M.D., F.E.S.C., ${ }^{*}$ Funda Aybar, M.D., $\dagger$ Giray Kabakci, M.D., F.E.S.C., \\ $\ddagger$ Hakan Yarali, M.D., $†$ and Ali Oto, M.D., F.A.C.C., F.E.S.C. $\ddagger$
}

From the *Department of Cardiology, Baskent University, †Departments of Obstetrics/Gynecology, and $\ddagger$ Department of Cardiology, Hacettepe University, Ankara, Turkey

\begin{abstract}
Background: Limited data are available related to the effects of sex hormones on cardiac autonomic function. Few studies investigated the heart rate variability (HRV) parameters during regular menstrual cycle or in postmenopausal women using hormone replacement therapy, but the results were contradictory. The aim of the study was to compare the characteristics of the autonomic innervation of the heart in polycystic ovary syndrome (PCOS) patients with regularly cycling controls.

Methods: Thirty PCOS patients and 30 healthy regularly cycling controls were included in the study. Groups were compared with respect to age and various cardiovascular risk factors. Characteristics of autonomic innervation of the heart were evaluated with HRV. Power spectral analysis of HRV was performed to calculate the low frequency peak (LF $0.04-0.15 \mathrm{~Hz}$ ), high-frequency peak (HF 0.15-0.40 Hz), LF in normalized unit (LF nu), HF in normalized unit (HF nu) and LF/HF ratio.

Results: PCOS patients had adverse cardiovascular risk profile than controls. As the HRV parameters, PCOS patients had significantly higher LF nu $(P=0.005)$ and LF/HF ratio $(P=0.001)$ and significantly lower HF $(P=0.006)$ and HF nu $(P<0.001)$ compared to controls.

Conclusion: Autonomic innervation of the heart can be affected in PCOS with increased sympathetic and decreased parasympathetic components of HRV. As a result, sympathetic to parasympathetic ratio may increase in PCOS. This finding should be confirmed with larger studies also evaluating the clinical implications of altered HRV parameters.

A.N.E. 2006;11(4):306-312
\end{abstract}

$\underline{\text { polycystic ovary syndrome; cardiac autonomic innervation; heart rate variability }}$

Polycystic ovary syndrome (PCOS) is a common endocrine disorder, affecting $6-10 \%$ of reproductive aged women. ${ }^{1}$ It not only is a reproductive endocrinopathy but, like the metabolic syndrome, is associated with long-term health risks including insulin resistance, diabetes mellitus, dyslipidemia, hypertension, and premature atherosclerosis. ${ }^{2}$ Based on the prevalence of these risk factors, PCOS patients have an estimated 4-11-fold increased risk for coronary heart disease. ${ }^{3}$

Several other aspects of cardiovascular involvement have also been previously investigated and many of them reported some abnormalities in PCOS patients compared to controls. These abnormalities include decreased cardiac systolic flow velocity, ${ }^{4}$ diastolic dysfunction, ${ }^{5}$ increased vas- cular stiffness, ${ }^{6}$ endothelial dysfunction ${ }^{7}$ lowgrade chronic inflammation, ${ }^{8}$ increased homocysteine, ${ }^{5,9}$ impaired fibrinolysis, ${ }^{10}$ and increased tissue plasminogen activator antigen. ${ }^{11}$ However, there is no study, reported so far, investigating the cardiac autonomic innervation in women with PCOS.

Heart rate variability (HRV) measurement from electrocardiographic recordings has been shown to be useful to assess cardiac autonomic function. ${ }^{12,13}$ The frequency-specific fluctuations in heart rate behavior and the sympathetic/parasympathetic integrity can be evaluated noninvasively with power spectrum analysis of HRV. The high-frequency component (HF $0.15-0.40 \mathrm{~Hz}$ ) represents vagal control of heart rate, whereas low-frequency component (LF $0.04-0.15 \mathrm{~Hz}$ ) and the ratio of LF to HF

Address for reprints: Aylin Yildirir, M.D., F.E.S.C., Associate Professor of Cardiology, Simon Bolivar Cad. No:18/34 Cankaya 06550 Ankara, Turkey. Fax: +90 31244135 53; E-mail: ayliny@baskent-ank.edu.tr 
represent sympathetic modulations or sympathovagal balance. ${ }^{12,13}$

The effects of menstrual cycle and hormone replacement therapy (HRT) on HRV have previously been investigated with contradictory results. ${ }^{14-31}$ To the best of our knowledge, no data are available on the characteristics HRV parameters in PCOS patients. Therefore, we aimed to investigate the characteristics of cardiac autonomic innervation with HRV in PCOS patients and compare them with healthy controls.

\section{METHODS}

\section{Study Population}

The study group consisted of 30 women who presented to the Obstetrics and Gynecology Clinic of Hacettepe University hospital with the complaints of dysfunctional uterine bleeding, hyperandrogenism or infertility and diagnosed to have PCOS. Diagnosis of PCOS was made with physical and laboratory findings of hirsutism or biochemical hyperandrogenism and chronic anovulation after exclusion of specific ovarian, adrenal, and pituitary disorders. ${ }^{32}$ Thirty healthy, regularly cycling (every 27-32 days) volunteer medical students, doctors or nurses served as the control group.

Exclusion criteria for both groups included pregnancy or lactation, diabetes mellitus, current use of lipid-lowering medications, oral hypoglycemic or insulin-sensitizing agents, oral contraceptives, or sex steroids; current infertility treatment; hysterectomy or menopause. All PCOS patients and control subjects were apparently in good health, free of systemic hypertension, renal disease, chronic lung disease, or overt heart disease.

The subjects included in the study were questioned about symptoms of autonomic nervous system dysfunction and underwent complete cardiac and neurologic examination. Those with abnormal cardiac and neurological examination were excluded. Baseline electrocardiograms (ECG) were obtained from all subjects and those with abnormal baseline ECG (including juvenile pattern) were also excluded.

All patients and controls gave informed consent for the study. A venous blood sample was taken after at least 12 hours fasting for the biochemical and hormonal analysis. Control subjects were in their follicular phase (6-8 days after the start of menses) and PCOS patients were amenorrheic during data recording for HRV.

\section{HRV Analysis}

Recordings for HRV analysis were made with a PC-based high resolution ECG system (Kardiosis, Ankara, Turkey) after 30 minutes resting in a silent environment in supine position. Bipolar X deviation $(0.5-340 \mathrm{~Hz})$ was recorded and sampled at a rate of 1,000 samples per second and digitized using an 8-bit A/D converter. Each recording lasted 8 minutes, and raw data were stored on disk for postprocessing. A segment of 5 minutes of the recording with no artifacts and premature depolarizations was processed to provide the results of HRV analysis. The RR tachograms were extracted from the data. The detection of $\mathrm{R}$ waves was done with a thresholding algorithm. The detected $\mathrm{R}$ waves were visually confirmed and any undetected regular $R$ wave was marked either manually or by interpolation. Similarly, any point, which is not, an R wave but found as one, was unmarked manually. By this method, raw RR tachograms were extracted. Raw tachogram was then interpolated at 1-second intervals by linear interpolation. From the interpolated RR tachogram, power spectral densities were calculated by autoregressive modeling. Power spectra for heart rate variations were calculated with autoregressive modeling. In power spectra of RR intervals, three major peaks, one around $0.03 \mathrm{~Hz}$ (very low frequency-VLF peak), one around 0.03$0.15 \mathrm{~Hz}$ (low frequency-LF peak) and the other around $0.15-0.40 \mathrm{~Hz}$ (High frequency-HF peak) were seen. The peak around $<0.03 \mathrm{~Hz}$ was attributed to several physiologic variables and was filtered out from the tachograms before modeling. For spectral analysis, the following variables were calculated: (1) The low-frequency component in absolute (LF) and normalized units (LF nu), (2) the high-frequency component in absolute (HF) and normalized units (HF nu), (3) the ratio of the absolute low- and high-frequency component (LF/HF). The absolute values of the results were in milliseconds for the time domain and milliseconds ${ }^{2}$ for power spectral analysis.

The power spectral analysis method of HRV from short-term recordings has been accepted as a reliable technique to evaluate the ANS functions ${ }^{12,13}$ and we used the same method in our previously published studies. ${ }^{19,31,33}$

\section{Statistical Analysis}

The Statistical Package for the Social Science (SPSS 9.1 version for Windows) was used for 
statistical analyses. The Kolmogorov-Smirnov goodness-of-fit test was used to test normality. Because of skewed distribution, LF power, HF power, plasma estradiol and testosterone levels were transformed by calculating their natural logarithm. Independent sample $t$-test was used to compare the measured parameters of patients with PCOS and control groups. Spearman correlation analyses were used to investigate the relationships between sex hormones and HRV parameters. Values were expressed as mean \pm SD. Statistical significance was set at $\mathrm{P}<0.05$.

\section{RESULTS}

The baseline clinical characteristics of PCOS patients and controls are given in Table 1 . Both groups included young subjects with a mean age of around 30 years, the PCOS group being younger than controls $(27.9 \pm 6.1$ vs $31.4 \pm 6.5, \mathrm{P}=0.034)$. The two groups were matched for body mass index, heart rate, systolic, and diastolic blood pressure values (all $\mathrm{P}>0.05$ ).

The mean fasting total cholesterol (T-C) and low density lipoprotein cholesterol (LDL-C) levels were comparable between the two groups. However, mean triglyceride level and T-C/high density lipoprotein cholesterol (HDL-C) ratio were significantly higher and HDL-C was significantly lower in the PCOS group (Table 1).
As the hormone levels of the study population are concerned, plasma follicle-stimulating hormone (FSH), luteinizing hormone (LH) and dehydroepiandrosterone sulfate (DHEAS) levels of PCOS patients were comparable with those of control subjects (all $\mathrm{P}>0.05)$. However, PCOS patients had significantly lower estradiol $(P=0.015)$ and borderline higher $(\mathrm{P}=0.051)$ testosterone levels than controls (Table 1).

The spectral analysis of HRV parameters of PCOS and control groups is given in Table 2. Power of LF was similar in both groups; however, PCOS patients had significantly higher LF nu than controls $(\mathrm{P}=0.005)$. On the other hand, power of $\mathrm{HF}$ and HF nu was significantly increased in controls compared to patients with PCOS $(\mathrm{P}=0.006$ and $\mathrm{P}<0.001$ respectively). Therefore, LF/HF ratio, was significantly higher in PCOS patients indicating an increased sympathetic activity $(\mathrm{P}=0.001)$.

Correlation analyses between HRV parameters and sex hormones of the study population $(n=60)$ are shown in Table 3. As indicated in the table, there was no significant correlation between HRV parameters and $\mathrm{FSH}, \mathrm{LH}$, estradiol, testosterone, and DHEAS levels. However, a tendency for a negative correlation between estradiol levels and LF/HF $(\mathrm{r}=-0.253, \mathrm{P}=0.060)$ and a tendency for a positive correlation between testosterone levels and LF $(\mathrm{r}=0.238, \mathrm{P}=0.086)$ can be noticed.

Table 1. Baseline Clinical Features of PCOS Patients and Controls

\begin{tabular}{lccc}
\hline & PCOS $(\mathbf{n}=\mathbf{3 0})$ & Control $(\mathbf{n}=\mathbf{3 0})$ & $\mathbf{P}$ \\
\hline Age $(\mathrm{y})$ & $27.9 \pm 6.1$ & $31.4 \pm 6.5$ & 0.034 \\
BMI $\left(\mathrm{kg} / \mathrm{m}^{2}\right)$ & $27.3 \pm 6.0$ & $25.0 \pm 3.3$ & 0.097 \\
Heart rate (beats/min) & $77.6 \pm 14.2$ & $73.4 \pm 9.1$ & 0.214 \\
Systolic BP (mmHg) & $121.7 \pm 16.4$ & $118.7 \pm 15.3$ & 0.376 \\
Diastolic BP (mmHg) & $81.2 \pm 23.5$ & $79.3 \pm 22.7$ & 0.420 \\
T-C (mg/dL) & $181.6 \pm 25.1$ & $171.9 \pm 35.0$ & 0.238 \\
LDL-C (mg/dL) & $107.3 \pm 24.2$ & $100.5 \pm 33.0$ & 0.379 \\
HDL-C (mg/dL) & $48.2 \pm 12.7$ & $55.2 \pm 10.5$ & 0.030 \\
Triglyceride (mg/dL) & $128.9 \pm 86.4$ & $81.2 \pm 47.6$ & 0.013 \\
T-C/HDL-C & $3.9 \pm 45.6$ & $3.2 \pm 0.9$ & 0.005 \\
FSH (IU/L) & $4.8 \pm 1.7$ & $6.1 \pm 3.7$ & 0.096 \\
LH (IU/L) & $7.3 \pm 4.1$ & $6.3 \pm 6.9$ & 0.510 \\
Estradiol (pg/mL) & $56.8 \pm 62.7$ & $104.9 \pm 91.1$ & 0.015 \\
Testosterone $(\mathrm{ng} / \mathrm{dL})^{a}$ & $93.2 \pm 63.3$ & $65.5 \pm 57.5$ & 0.051 \\
DHEAS (micg/dL) & $198.1 \pm 102.0$ & $153.8 \pm 79.6$ & 0.074 \\
\hline
\end{tabular}

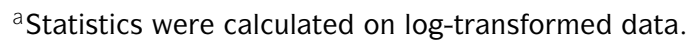

$\mathrm{PCOS}=$ polycystic ovary syndrome; $\mathrm{BMI}=$ body mass index; $\mathrm{BP}=$ blood pressure, $\mathrm{T}-\mathrm{C}=$ total cholesterol; LDL-C = low density lipoprotein cholesterol; $\mathrm{HDL}-\mathrm{C}=$ high density lipoprotein cholesterol; FSH = follicle-stimulating hormone; $\mathrm{LH}=$ luteinizing hormone, DHEAS = dehydroepiandrosterone sulfate. 
Table 2. HRV Measures of PCOS Patients and Controls

\begin{tabular}{lccr}
\hline & PCOS $(\mathbf{n}=\mathbf{3 0})$ & Control $(\mathbf{n}=\mathbf{3 0})$ & P \\
\hline $\mathrm{LF}\left(\mathrm{ms}^{2} / \mathrm{Hz}\right)^{\mathrm{a}}$ & $434 \pm 454$ & $440 \pm 311$ & 0.830 \\
$\mathrm{LF} \mathrm{nu}$ & $59 \pm 20$ & $46 \pm 10$ & 0.005 \\
$\mathrm{HF}\left(\mathrm{ms}^{2} / \mathrm{Hz}\right)^{\mathrm{a}}$ & $411 \pm 933$ & $488 \pm 425$ & 0.006 \\
$\mathrm{HF}$ nu & $34 \pm 10$ & $45 \pm 11$ & $<0.001$ \\
LF/HF & $1.8 \pm 0.8$ & $1.1 \pm 0.6$ & 0.001 \\
\hline
\end{tabular}

a Statistics were calculated on log-transformed data.

$\mathrm{PCOS}=$ polycystic ovary syndrome; LF = low frequency; HF = high frequency; LF nu = low frequency in normalized unit; HF nu = high frequency in normalized unit; LF/HF = low frequency/high frequency ratio.

\section{DISCUSSION}

HRV is a statistical measure of the cyclic beatto-beat variation of heart rate, which correlates with the individual autonomic tone and is used to quantify risk in a wide variety of both cardiac and noncardiac disorders. These include stroke, multiple sclerosis, end-stage renal disease, neonatal distress, diabetes mellitus, ischemic heart disease, cardiomyopathy, valvular heart disease, and congestive heart failure. ${ }^{13} \mathrm{HRV}$ analysis has also been used to assess the autonomic effects of various drugs including beta-blockers, calcium channel blockers, antiarrhythmics, psychotropic agents, and cardiac glycosides. ${ }^{13}$ Diminished HRV is associated with increased sympathetic and decreased vagal modulation, and these autonomic changes have been reported to be associated with an increase in malignant ventricular arrhythmias. ${ }^{12,13}$ The greatest potential therapeutic use of HRV analysis is in the risk stratification of patients after myocardial infarction. ${ }^{34,35}$

Other than several disorders and drugs some physiologic conditions also alter HRV. Previous research indicates that age is a major determinant of HRV, and that HRV decreases with increasing age. ${ }^{36,37}$ Gonadal hormones, both androgens and estrogens, may influence autonomic nervous system function, thus HRV. Previous studies report sex-related differences in HRV. ${ }^{15,36,37}$ Data related to the effects of sex steroids on HRV are mainly based on studies related to postmenopausal HRT $^{14-25}$ or those evaluating autonomic nervous system functions in different phases of menstrual cycle. ${ }^{26-31}$

Premenopausal women are reported to have higher HRV than postmenopausal women, ${ }^{38}$ which suggests that fluctuations in endogenous sex hormones during menopause may play a role in agerelated differences in HRV. This finding has led researchers to speculate that postmenopausal HRT may influence autonomic nervous system functions. Most of the studies evaluating the effects of HRT on HRV reported favorable results. ${ }^{14-19}$ Rosane et al. ${ }^{14}$ reported decreased ratio of $\mathrm{LF}$ and HF power after 4 months of estrogen use. Huikuri et al. ${ }^{15}$ indicated that postmenopausal women who used estrogen replacement therapy had higher HRV (lower LF and higher HF) than age-matched nonusers. Mercuro et al. ${ }^{16}$ reported decreased parasympathetic modulation and a shift of autonomic cardiovascular control toward sympathetic hyperactivity after oophorectomy and in this study estrogen replacement therapy returned HRV

Table 3. Results of Correlation Analyses between Sex Hormones and Heart Rate Variability Parameters of the Study Population

\begin{tabular}{lllllllll}
\hline & Heart rate $(\mathbf{n}=\mathbf{6 0})$ & \multicolumn{2}{c}{ LF $(\mathbf{n}=\mathbf{6 0})$} & HF $(\mathbf{n}=\mathbf{6 0})$ & LF/HF ratio $(\mathbf{n}=\mathbf{6 0})$ \\
\hline FSH & $\mathrm{r}=0.123$ & $\mathrm{P}=0.367$ & $\mathrm{r}=-0.066$ & $\mathrm{P}=0.631$ & $\mathrm{r}=0.055$ & $\mathrm{P}=0.688$ & $\mathrm{r}=-0.182$ & $\mathrm{P}=0.179$ \\
LH & $\mathrm{r}=0.149$ & $\mathrm{P}=0.274$ & $\mathrm{r}=0.105$ & $\mathrm{P}=0.440$ & $\mathrm{r}=0.156$ & $\mathrm{P}=0.250$ & $\mathrm{r}=-0.010$ & $\mathrm{P}=0.941$ \\
Estradiol & $\mathrm{r}=-0.93$ & $\mathrm{P}=0.495$ & $\mathrm{r}=-0.212$ & $\mathrm{P}=0.117$ & $\mathrm{r}=0.179$ & $\mathrm{P}=0.187$ & $\mathrm{r}=-0.253$ & $\mathrm{P}=0.060$ \\
Testosterone & $\mathrm{r}=0.230$ & $\mathrm{P}=0.097$ & $\mathrm{r}=0.238$ & $\mathrm{P}=0.086$ & $\mathrm{r}=0.079$ & $\mathrm{P}=0.574$ & $\mathrm{r}=0.144$ & $\mathrm{P}=0.303$ \\
DHEAS & $\mathrm{r}=0.149$ & $\mathrm{P}=0.267$ & $\mathrm{r}=0.222$ & $\mathrm{P}=0.097$ & $\mathrm{r}=0.028$ & $\mathrm{P}=0.837$ & $\mathrm{r}=0.141$ & $\mathrm{P}=0.297$ \\
\hline
\end{tabular}

$\mathrm{LF}=$ Iow frequency; HF = high frequency; LF/HF = low frequency/high frequency ratio; FSH = follicle-stimulating hormone; $\mathrm{LH}=$ luteinizing hormone; DHEAS = dehydroepiandrosterone sulfate. 
parameters to baseline in 3 months. Another study on postmenopausal hypertensive women reported improved 24-hour indices of HRV with estrogen replacement therapy..$^{17}$ Liu et al. ${ }^{18}$ indicated reduced vagal and increased sympathetic indices of HRV in postmenopausal women compared with those of the premenopausal women. Our study group ${ }^{19}$ in a previous report, evaluated the effects of a 6month course of HRT (estrogen alone or combined with progresteron) on various HRV indexes on 46 women and reported significantly decreased LF/HF and increased HF power after 6 months of HRT.

There are also studies reporting different effects of estrogen versus estrogen/progesterone replacement therapy on HRV. Gökce et al. ${ }^{20}$ reported that a long-term (1 year) use of estrogen replacement therapy in postmenopausal healthy women increased the HRV indices of parasympathetic activity, and decreased the HRV indices of sympathetic activity. However, progesterone/estrogen replacement therapy did not affect both parasympathetic and sympathetic components of HRV.

Contrary to the studies, mentioned above, indicating favorable effects of HRT (mainly estrogen) on HRV, there are also studies reporting either null $^{21-21}$ or unfavorable ${ }^{25}$ results. A large longterm cohort study of 2621 women found no association between HRT and HRV. ${ }^{21}$ In another trial of healthy postmenopausal women, Farag et al, ${ }_{1}^{22}$ found no significant effect of an estrogen-only replacement regimen on HRV. Fernandes et al. ${ }^{23}$ who compared two different forms of continuous hormone replacement regimens, estradiol alone, and estradiol plus norethisterone found no significant effect of HRT on 24-hour time-domain indices of HRV. In addition to those, Virtanen et $\mathrm{al}^{24}$ reported no changes in the frequency- or time-domain analysis of HRV parameters after 3 months of estradiol replacement therapy.

In contrast to favorable or null results, Christ et $\mathrm{al}_{1}{ }^{25}$ in a cross-sectional study reported that progesterone-containing replacement therapy, but not estrogen-only program, was associated with an attenuation of HRV. Therefore, part of the controversy on the effects of HRT on autonomic modulation may be related to the different methodologies in HRV analysis, and the other part most probably to the differences in drug regimens. If we globally evaluate all above-mentioned studies, estrogen seems to have more favorable effect on HRV than progesterone alone or the combined regimen.
The other main study group to evaluate the effects of sex hormones on cardiac autonomic function is regularly cycling women, follicular phase with estrogen dominance and luteal phase with progesterone dominance. ${ }^{27-31}$ Goldstein et al ${ }^{26}$ reported increased plasma catecholamine levels in the luteal phase compared to follicular phase suggesting alteration in the cardiac autonomic innervation during different phases of menstrual cycle. Sato et al. ${ }^{27}$ investigated the fluctuations of autonomic nervous system activities during the menstrual cycle and reported increased LF component and LF/HF ratio and decreased HF component of HRV during the luteal phase. Similarly, Guasti et al. ${ }^{28}$ reported significantly higher LF/HF ratio and LF nu and lower HF nu in the luteal phase of the menstrual cycle, indicating a relatively higher sympathetic activity during this phase. In another study, Saeki et al ${ }^{29}$ found that parasympathetic activity is predominant in the follicular phase. Contradictory to these studies Fuenmayor et al. ${ }^{30}$ reported significantly higher Valsalva index during the luteal phase compared to follicular phase suggesting an increase in parasympathetic activity during the luteal phase of menstrual cycle. However, this study was limited in methodology since, it investigated the Valsalva index, which was a crude method for the evaluation of autonomic nervous system functions rather than computer-based power spectral analysis of HRV. Our study group ${ }^{31}$ also previously investigated the effects of menstrual cycle on HRV and reported significantly increased LF nu and LF/HF ratio in the luteal phase compared to follicular phase, suggesting increased sympathetic activity in the luteal phase.

PCOS is a disorder of chronic anovulation, hyperandrogenism, hirsutism, obesity, subfertility, and insulin resistance. Despite the presence of several well-documented risk factors, cardiac involvement in this syndrome has not been investigated thoroughly and no data are available related to the cardiac autonomic nervous system functions in PCOS. Therefore, in this study we evaluated the involvement of cardiac autonomic innervation in young women with PCOS and compared our results with regularly cycling controls. Our results indicated that PCOS patients had significantly higher LF nu $(\mathrm{P}=0.005)$ and $\mathrm{LF} / \mathrm{HF}$ ratio $(\mathrm{P}=0.001)$ than controls; whereas, power of $\mathrm{HF}$ and $\mathrm{HF}$ nu was significantly higher in controls than PCOS patients ( $\mathrm{P}=0.006$ and $\mathrm{P}<0.001$, respectively). Therefore, PCOS patients had an unfavorable cardiac 
autonomic innervations profile, with an increased sympathetic activity, compared to regularly cycling women. Differences in the hormonal profile of both groups might be responsible from this result. Although, as mentioned above, the data related to the effects of sex hormones on HRV are contradictory ${ }^{14-31}$ studies reporting favorable effects of estrogen replacement in postmenopausal period ${ }^{14-19}$ or more favorable HRV parameters in the estrogen predominant phase (follicular) during menstrual cycle $27-29,31$ are more consistent than others. Therefore, these unfavorable HRV parameters in the PCOS patients might be related to significantly lower plasma estradiol $(\mathrm{P}=0.015)$ and borderline higher plasma testosterone levels $(\mathrm{P}=0.051)$ in this group compared to control subjects. Although, the correlation analyses between sex hormones and HRV parameters of the study population failed to show significant associations, the tendencies for a positive correlation between testosterone levels and LF $(\mathrm{P}=0.086)$ and for a negative correlation between estradiol levels and LF/HF $(\mathrm{P}=0.060)$ deserve special attention. Therefore, despite the insignificant results of the correlation analyses, the potential role of the differences in sex hormones for the alterations in HRV parameters in PCOS cannot be excluded.

In studying the autonomic nervous system, various techniques and maneuvers can be used to detect the function or integrity of the sympathetic and parasympathetic components, including cold pressor test, Valsalva maneuver, tilt table testing, etc. $\mathrm{HRV}$ is a noninvasive study procedure utilizing spontaneous fluctuations in heart rate to estimate tonic functions of the autonomic nervous system and requires a well-controlled condition for recordings. In the present study, we used spectral analysis of HRV while subjects were in supine rest in a quiet and relaxing atmosphere. In addition, due to the skewness of the distribution of the data logarithmic transformation was used for HF and LF measures. The recording of spontaneous heart rate signals, the control of the experimental environment, and the correction for skewness of the HRV indices in our study may offer a reliable estimation of tonic autonomic nervous system functions. We used the same methodology in our previously published studies related to the effects of sex hormones on HRV. ${ }^{19,31}$

Although, it was our intention to match the mean age of the PCOS patients and control subjects, we ended up with statistically significant mean younger age in the PCOS patients. Because, HRV may worsen with aging ${ }_{1}^{36,37}$ a significant younger mean age of the PCOS patients in our study may have resulted in underestimation of our results, rather than creating a contradiction. Furthermore, a difference of about 3.5 years in the mean ages of the two groups may also not be clinically significant.

Our results must be interpreted in the light of some limitations. One of the main limitations is the small sample size of both study groups and the other is the lack of clinical data about the prevalence of arrhythmias.

Despite these limitations, to our knowledge, this is the first study to investigate the cardiac autonomic innervation in patients with PCOS by means of HRV parameters. The results of the present study show that cardiac autonomic innervation can be affected in PCOS with increased sympathetic and decreased parasympathetic components of HRV. As a result, sympathetic to parasympathetic ratio may increase in PCOS. In the light of this data, further research with larger sample size is now warranted to evaluate the HRV measures and their clinical significance in this special group of young women with PCOS.

\section{REFERENCES}

1. Carmina E, Lobo RA. Polycystic ovary syndrome (PCOS): Arguably the most common endocrinopahy is associated with significant morbidity in women. J Clin Endocrinol Metab 1999;84:1897-1899.

2. Legro RS. Polycystic ovary syndrome and cardiovascular disease: A premature association? Endo Rev 2003;24:302312.

3. Dahlgren E, Janson PO, Johansson S, et al. Polycystic ovary syndrome and risk for myocardial infarction: Evaluated from a risk factor model based on a prospective population study of women. Acta Obstet Gynecol Scand 1992;71:599604.

4. Prelevic GM, Beljic T, Balint-Peric $\mathrm{L}$, et al. Cardiac flow velocity in women with polycystic ovary syndrome. Clin Endocrinol 1995:43:677-681.

5. Yarali H, Yildirir A, Aybar F, et al. Diastolic dysfunction and increased serum homocysteine concentrations may contribute to increased cardiovascular risk in patients with polycystic ovary syndrome. Fertil Steril 2001;76:511-516.

6. Kelly CJ, Speirs A, Gould GW, et al. Altered vascular function in young women with polycystic ovary syndrome. J Clin Endocrinol Metab 2002;87:742-746.

7. Paradisi G, Steinberg HO, Hempfling A, et al. Polycystic ovary syndrome is associated with endothelial dysfunction. Circulation 2001;103:1410-1415.

8. Kelly CC, Lyall H, Petrie JR, et al. Low grade chronic inflammation in women with polycystic ovary syndrome. J Clin Endocrinol Metab 2001;86:2453-2455.

9. Loverro G, Lorusso F, Mei L, et al. The plasma homocysteine levels are increased in polycystic ovary syndrome. Gynecol Obstet Invest 2002;53:157-162. 
10. Yildiz BO, Haznedaroglu IC, Kirazli S, et al. Global fibrinolytic capacity is decreased in polycystic ovary syndrome, suggesting a prothrombotic state. J Clin Endocrinol Metab 2002;87:3871-3875.

11. Kelly CJ, Lyall $H$, Petrie JR, et al. A specific elevation in tissue plasminogen activator antigen in women with polycystic ovary syndrome. J Clin Endocrinol Metab 2002;87:3287-3290.

12. Task Force of the European Society of Cardiology and the North American Society of Pacing and Electrophysiology. Heart rate variability. Standards of measurement, physiologic interpretation, and clinical use. Circulation 1996;93:1043-1065.

13. Kleiger RE, Stein PK, Bigger JT. Heart rate variability: Measurement and clinical utility. Ann Noninvasive Electrocardiol 2005;10:88-101.

14. Rosano GMC, Patrizi R, Leonardo F, et al. Effect of estrogen replacement therapy on heart rate variability in healthy postmenopausal women. Am J Cardiol 1997;80:815-817.

15. Huikuri HV, Pikkujamsa SM, Airaksinen KEJ, et al. Sexrelated differences in autonomic modulation of heart rate in middle-aged subjects. Circulation 1996;94:122-125.

16. Mercuro $G$, Longu $G$, Zoncu $S$, et al. Impaired forearm blood flow and vasodilator reserve in healthy postmenopausal women. Am Heart J 1999;137:692-697.

17. Brito-Zurita OR, Posadas-Romero C, Hermosillo A, et al. Estrogen effect on heart rate variability in hypertensive postmenopausal women. Maturitas 2003;44:39-48.

18. Liu CC, Kuo TB, Yang CC. Effects of estrogen on genderrelated autonomic differences in humans. Am J Physiol Heart Circ Physiol 2003;56:565-571.

19. Yildirir A, Kabakci G, Yarali $H$, et al. Effects of hormone replacement therapy on heart rate variability in postmenopausal women. Ann Noninvasive Electrocardiol 2001;6:280-284.

20. Gokce M, Karahan B, Yilmaz R, et al. Long term effects of hormone replacement therapy on heart rate variability, QT interval, QT dispersion and frequencies of arrhytmia. Int J Cardiol 2005;99:373-379.

21. Carnethon MR, Anthony MS, Cascio WE, et al. Prospective association between hormone replacement therapy, heart rate, and heart rate variability. The Atherosclerosis Risk in Communities Study. J Clin Epidemiol 2003;56:565-571.

22. Farag $\mathrm{NH}$, Nelesen RA, Parry BL, et al. Autonomic and cardiovascular function in postmenopausal women: The effects of estrogen versus combination therapy. Am J Obstet Gynecol 2002;186:954-961.

23. Fernandes EO, Moraes RS, Ferlin EL, et al. Hormone replacement therapy does not affect the 24-hour heart rate variability in postmenopausal women. Pacing Clin Electrophysiol 2005;28:S172-S177.
24. Virtanen I, Polo-Kantola P, Erkkola R, et al. Climacteric vasomotor symptoms do not imply autonomic dysfunction. Br J Obstet Gynecol 1999;106:155-164.

25. Christ $M$, Seyffart $K$, Wehling $M$. Attenuation of heart-rate variability in posmenopausal women on progestin-containing hormone replacement therapy. Lancet 1999;353:1939-1940.

26. Goldstein DS, Levinson P, Keiser HR. Plasma and urinary catecholamines during the human ovulatory cycle. J Obstet Gynecol 1983;146:824-829.

27. Sato N, Miyake S, Akatsu J, et al. Power spectral analysis of heart rate variability in healthy young women during the normal menstrual cycle. Psychosom Med 1995;57:331-335.

28. Guasti L, Grimoldi P, Mainardi LT, et al. Autonomic function and baroreflex sensitivity during a normal ovulatory cycle. Acta Cardiol 1999;54:209-213.

29. Saeki Y, Atogami F, Takahashi K, et al. Reflex control of autonomic function induced by posture change during the menstrual cycle. J Auton Nerv Syst 1997;66:69-74.

30. Fuenmayor AJ, Ramirez L, Fuenmayor AM. Left ventricular function and autonomic nervous system balance during two different stages of the menstrual cycle. Int J Cardiol 2000;72:243-246.

31. Yildirir A, Kabakci G, Akgul E, et al. Effects of menstrual cycle on cardiac autonomic innervation as assessed by heart rate variability. Ann Noninvasive Electrocardiol 2002;7:6063.

32. Dunaif A. Hyperandrogenic anovulation (PCOS): A unique disorder of insulin action associated with an increased risk of non-insulin-dependent diabetes mellitus. Am J Med 1995;98:33S-39S

33. Tokgozoglu SL, Batur MK, Topcuoglu MA, et al. Effects of stroke localization on cardiac autonomic balance and sudden death. Stroke 1999:30:1307-1311.

34. Fei L, Copie $X$, Malik M, et al. Short- and long-term assessment of heart rate variability for risk stratification after acute myocardial infarction. Am J Cardiol 1996;77:681684.

35. Quintana M, Storck N, Lindblad LE, et al. Heart rate variability as a means of assessing prognosis after acute myocardial infarction. A 3-year follow-up study. Eur Heart J 1994:18:789-797.

36. Liao D, Barnes RW, Chambless LE, et al. Age, race, and sex differences in autonomic cardiac function measured by spectral analysis of heart rate variability-The ARIC study. Am J Cardiol 1995;76:906-912.

37. Tsuji H, Ferdinand J, Venditti J, et al. Determinants of heart rate variability. J Am Coll Cardiol 1996;28:1539-1546.

38. Brockbank CL, Chatterjee F, Bruce SA, et al. Heart rate and its variability change after the menopause. Exp Physiol 2000;85:327-330 